\title{
Deformation and energy of dry snow slabs prior to fracture propagation
}

\author{
D.M. MCCLUNG, ${ }^{1}$ C.P. BORSTAD ${ }^{2}$ \\ ${ }^{1}$ Department of Geography, University of British Columbia, Vancouver, British Columbia, Canada \\ E-mail: mcclung@geog.ubc.ca \\ ${ }^{2}$ Department of Civil Engineering, University of British Columbia, Vancouver, British Columbia, Canada
}

\begin{abstract}
Snow slab avalanches primarily release by propagation of shear fractures within thin weak layers under thicker slabs. The weak layer is typically on the order of $\mathbf{1} \mathbf{~ m m}$ thick and fails in mode II. In some cases, the weak layer is thicker and there may be a need to consider slope-normal deformation as part of the energy condition prior to rapid propagation. In this paper, field measurements from shear fracture initiation and high-speed films are combined to consider the effects of slope-normal deformation on bending of the slab prior to propagation and its relation to the propagation condition. Slab bending is modelled using two limiting cases: (1) a uniformly loaded beam supported by a deforming weak layer, analogous to a Winkler foundation, and (2) a uniformly loaded unsupported cantilevered beam. The experimental and analytical results suggest that slab bending prior to fracture initiation is small or negligible. Two previous approaches to modelling slab avalanche initiation involving slab bending are discussed. Both models proposed strong slab-bending effects prior to initiation, which conflicts with our results. Our field observations and modelling both show that strong bending is a dynamic effect following slope-parallel weak-layer fracture initiation.
\end{abstract}

\section{INTRODUCTION}

The first mathematical model to describe the mechanics of dry snow slab-avalanche initiation was by Perla and LaChapelle (1970) and Perla (1971). They defined two separate avalanche initiation scenarios depending on weaklayer thickness. If the weak layer was very thin, they described the initiation process by shear failure within the weak layer. For thicker layers, they described the failure process in terms of collapse of the weak layer, which implied slope-normal deformation and bending of the slab. For weak layers of finite thickness, on the order of $\sim 10 \mathrm{~mm}$ or more, the collapse is often observed during dynamic slope-parallel fracture propagation in the weak layer even when the slope angle is zero. The drop associated with dynamic collapse can be sensed as noise from dynamic compression of the weaklayer air between the pore spaces. This is called a 'whumph' from its phonetic description. Since Perla's work, descriptions and modelling in regard to the collapse process (Johnson and others, 2004; Heierli and others, 2008) have largely been guided by descriptive field observations after propagation has begun, rather than by detailed observations about how alpine snow fails and fractures and measurements of deformation prior to fracture propagation. These earlier models have all placed bending within the slab as the primary source of stored energy in slab avalanche initiation when the weak layer has finite thickness. At present, the only quantitative models of bending or collapse in relation to avalanche initiation are those of Perla (1971) and Heierli and others (2008). After introducing our field observations and measurements, these two models are discussed and compared.

The stratigraphy appropriate to slab avalanche initiation is always characterized as a thick, cohesive planar slab on top of a relatively thin weak layer (Perla, 1971; McClung, 1979, 1981; Schweizer and others, 2003; McClung and Schaerer, 2006). In the present paper, slab deformation energy from slope-normal deformation is modelled assuming a stronger, cohesive slab supported on a weak deformable foundation to simulate the weak layer. The formalism contains the same assumptions as a Winkler (1867) foundation, which has found success in soil foundation work and for floating ice (Bažant and Cedolin, 2003). Other formulations exist for modelling the deformation for a beam on a deformable foundation, but the Winkler assumptions are the simplest. For performance in practical applications, the Winkler foundation is regarded as being as good as or better than more complex models (Bažant and Cedolin, 2003).

The Winkler foundation is used for three reasons: (1) It is the simplest known such foundation. The facts about alpine snow are not yet known well enough to justify a more complex model. (2) The field observations reported in this paper suggest that the deforming snow beams are in weak contact with the deforming layer, so some type of analysis with foundation support is needed. We also include the case of an unsupported beam to illustrate the case of maximum bending. (3) The main application of the results in this paper is to work toward an understanding of the conditions of dry snow slab-avalanche initiation. The fieldwork and observations reported below suggest that, in the case of avalanche release, the slab will be supported to some extent prior to avalanche initiation. Thus, the simpler case of an unsupported beam will likely not hold for explaining avalanche initiation.

The results, including field and laboratory measurements, field observations and modelling, all suggest a reversal of the concepts in the previous models. Namely, we propose that the effects of slab bending due to slope-normal deformation prior to initiation are small. When combined, the laboratory and field results suggest that the effects of slab bending prior to dynamic fracture propagation are very small or even negligible in terms of the propagation condition. The results justify the assumption (Palmer and Rice, 1973; McClung, 1979, 1981, 2009a, 2011) that, prior to propagation, the primary source of deformation is within the weak layer, not the slab. For a surface-hoar weak layer, the most common 


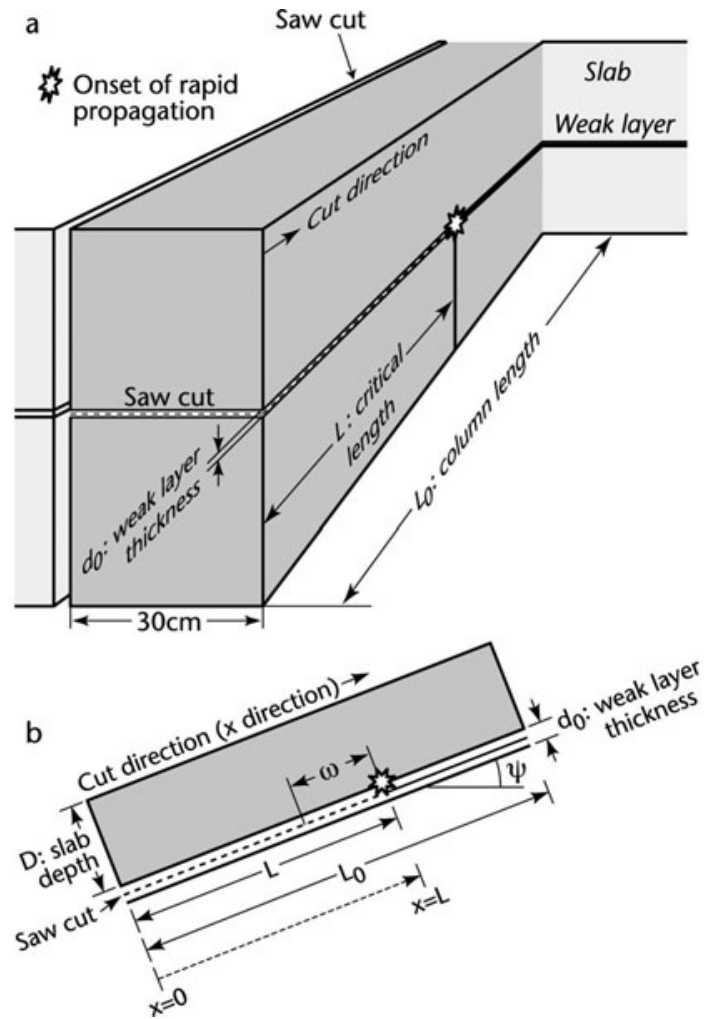

Fig. 1. (a) Schematic for field tests made with a saw-cut of critical length $L$ within a weak layer of thickness $d_{0}$ with a total block length $L_{0}$. (b) Side-view schematic of the field tests, with $D$ as the slab depth and slope angle $\psi$. The parameter $\omega$ is the length of the fracture process zone. The coordinate system is defined such that $x$ is measured from the left end of the block $(x=0)$ to the end of the cut or slip surface $(x=L)$ in the direction of the dotted line in the center of the weak layer.

weak layer in the field test data, this assumption has been confirmed from precision laboratory tests by Reiweger and Schweizer (2010).

Two goals are sought in this paper. The first is to discuss the previous models of Perla (1971) and Heierli and others (2008). The second is to present some simple data and analysis that may apply to avalanche initiation when considering the effects of slope-normal deformation.

\section{DATA AND OBSERVATIONS FROM FIELD EXPERIMENTS FROM PREVIOUS WORK}

All the field measurements in this paper are derived from experiments called propagation saw tests which involve making a saw-cut in a weak layer under a snow slab until slope-parallel weak-layer propagation is achieved. Sigrist (2006), Gauthier and Jamieson (2008) and McClung (2009a, 2011) provide detailed descriptions of these field tests. A brief description is given here. The procedure involves introducing a cut upslope within a weak layer underneath a long rectangular block of snow. The cut is made to a critical length, $L$, at which the slope-parallel fracture propagates rapidly within the weak layer. Figure 1 contains a schematic of the test set-up. The block of snow is $30 \mathrm{~cm}$ wide with a total length, $L_{0}$, ideally more than twice the depth, $D$. The standard length of the block is $1 \mathrm{~m}$, with the maximum cut length (the median of a series of tests for an individual layer) in all the tests as $\sim 65 \mathrm{~cm}$.
The values of $L$ used in this paper are median values derived from a series of tests, each of 68 slab-weak-layer combinations comprising 750 tests. Approximately $90 \%$ of the values come from Gauthier (2007) and Gauthier and Jamieson (2008).

In most tests, both the lower (downslope) and upper (upslope) ends of the block are cut in the slope-normal direction so that they represent free surfaces. Gauthier and Jamieson (2008) showed that there is no statistical difference between cut lengths made up- or downslope. For the measurements and observations described in the present paper, the cut is taken in the upslope direction unless otherwise stated. Given the standard $1 \mathrm{~m}$ block length and maximum cut length $(65 \mathrm{~cm})$ in the dataset, it is a good assumption that any free surface on the upslope end of the block does not affect the results.

In order to perform the tests, the weak layer must be sufficiently thick $(>5 \mathrm{~mm})$ that the snow saw can be moved through the weak layer without cutting into the slab. This is a limitation of the tests, in that some weak layers in slab avalanches are only on the order of $1 \mathrm{~mm}$ thick (e.g. stellar crystal layers) and it would not be possible to move the saw so as not to intersect the slab. Thus, the data used in this paper do not apply to weak layers less than $\sim 5 \mathrm{~mm}$.

\section{OBSERVATIONS AND DATA IN RELATION TO SAW TESTS}

In addition to the propagation saw test data, we observed and measured saw tests for developing weak-layer fractures and slab deformation using high-speed films (300 frames s ${ }^{-1}$ ) before, during and after fracture propagation in the weak layer in February 2009. The important results are listed below.

1. Strong bending and dynamic collapse are observed only after a fracture has propagated. Thus, strong bending and collapse are precipitated by a fracture.

2. Two types of fracture precede dynamic collapse and the associated strong bending: either a tensile fracture through the slab from top to bottom or a slope-parallel fracture through the weak layer. The type of initial fracture observed depends on the saw thickness used. For a $1 \mathrm{~mm}$ thick saw, slab tensile fractures are observed to occur much less frequently than for a $3 \mathrm{~mm}$ thick saw. We believe this effect is due to the larger gap or disturbance created by the thicker saw to produce more bending and tensile fracture. The volume fraction filled by air in the weak layer is typically $\sim 80 \%$. The disturbance caused by the saw is bound to produce some artificial deformation.

3. As the saw-cut progresses (Fig. 3, further below), the slab begins to bend slightly, with total slope-normal displacement of $\sim 1 \mathrm{~mm}$ (at the free end) over slope-parallel distances of saw-cut up to $1 \mathrm{~m}$. The weak-layer crystals remain in contact with the slab and the substratum during the cut.

4. The slope-normal deformation at the free surface end of the block is $\sim 1 \mathrm{~mm}$ prior to any propagating fracture or weak-layer collapse. Slope-normal deformations greater than $\sim 1 \mathrm{~mm}$ are observed only after dynamic weak-layer 
propagation or tensile fracture through the slab has caused dynamic collapse (see Section 3.1).

5. The saw tests may be envisioned as a notch cut in the weak layer but not a sharp crack with infinitesimal distance between the crack faces. The notch creates a gap in the weak layer, allowing the slab to sink into the weak layer and bend slightly as the cut is lengthened. Two extreme cases may be thought of in relation to modelling. One of these is to envision the slab as a simple cantilever beam which may or may not be supported by the weak layer. The other extreme is to think of the notch as equivalent to a sharp crack which is being closed as the crack lengthens and the slab bends. In reality, the saw tests are somewhere in between these extreme cases. Our modelling approach considers only the cantilever beam case since it implies the most bending. However, we have included comments about the fracture mechanical case.

\subsection{Results from high-speed films ( 300 frames $\mathrm{s}^{-1}$ )}

Figure 2 contains an example of the field measurements illustrated by tracking four particles in the slab. It shows slope-normal displacement, speed and acceleration of the particles. The total slope-normal displacement is slightly less than $1 \mathrm{~mm}$ prior to slope-parallel propagation. For Figure 2, $D=0.82 \mathrm{~m}, \psi=33^{\circ}, \rho=240 \mathrm{~kg} \mathrm{~m}^{-3}$ and $L=0.39 \mathrm{~m}$. For scale, $1 \mathrm{~s}$ represents about $10-20 \mathrm{~cm}$ of saw-cut. Thus, the slope-parallel scale of the cut $(20 \mathrm{~cm})$ over $1 \mathrm{~s}$ in Figure 2 is $\sim 200$ times the slope-normal displacement $(0.001 \mathrm{~m})$ when the slope-parallel propagation condition is met. It is of interest that the scale of slope-normal displacement $(0.001 \mathrm{~m}$ or less) was found in several films. All the information we have suggests that the slope-normal displacement, at propagation, is of order $1 \mathrm{~mm}$. These values suggest (but do not prove) that slope-normal displacement and bending effects are small prior to propagation. Roughly, if it is considered that the deformation takes place on the arc of a circle, simple geometry suggests that the radius of curvature (cut length $L=0.39 \mathrm{~m}$ ) at propagation is $R \approx L^{2} / 0.001=152 \mathrm{~m}$. In the following analysis, we consider this calculation in more detail using mechanical modelling of the bending process.

\subsection{Summary of information from the saw tests and information needed for estimating slab deformation}

The saw-test data include measurements of $L, D, \rho$ and $\psi$ for each test, where $\rho$ and $\psi$ are mean slab density and slope angle respectively. The dataset with all four parameters measured consists of 42 slab-weak-layer combinations comprising 559 individual tests.

In addition to these measurements, for the slab deformation calculations below, we need estimates of the effective viscoelastic slab modulus $E^{\prime}=2 \mu /(1-v)$, where $\mu$ is an effective shear modulus, which is rate- and density-dependent, and the effective Poisson's ratio, $v$, is taken as $v=0.1$ based on the estimates of Mellor (1975) and Salm (1977) for the low-density snow used in the tests $\left(\rho=85-266 \mathrm{~kg} \mathrm{~m}^{-3}\right)$.

The formulation we use for $E^{\prime}$ is equivalent to the assumption that the deformation is described by a twoparameter $(\mu, v)$ linear compressible stress-strain relation as with linear elasticity. The value of $E^{\prime}$ is specified as a material property appropriate for the deformation rate in the experiments and the slab density, both of which are known.
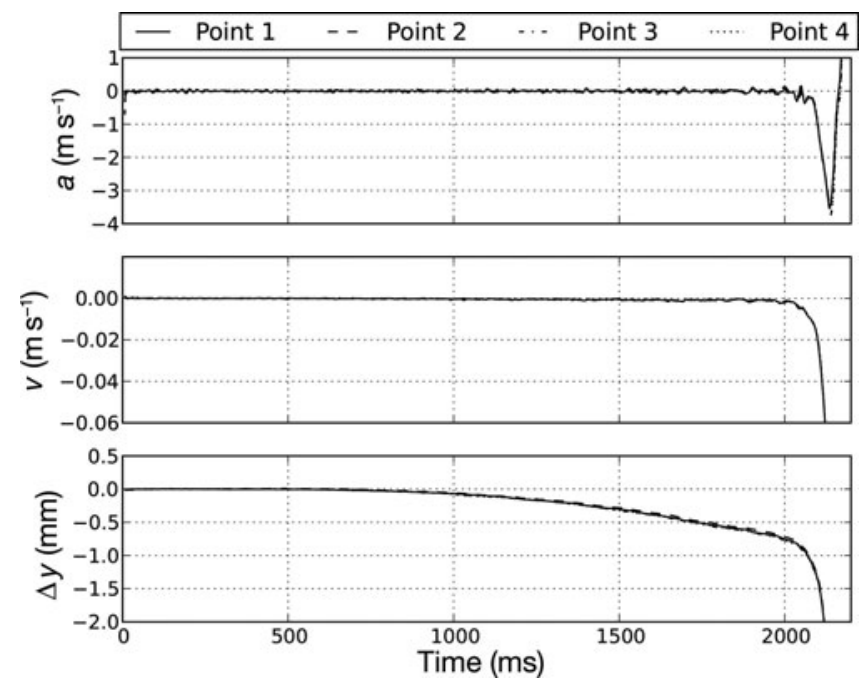

Fig. 2. Four particle traces within the slab for a propagation saw test (resolution $0.11 \mathrm{~mm}$ pixel $^{-1}$ ). The particles are located within the slab near the free end where the saw-cut was begun. Shown are the slope-normal acceleration, $a$, the slope-normal speed, $v$, and the slope-normal displacement, $\Delta y$. The slab thickness was $0.82 \mathrm{~m}$, $\rho=240 \mathrm{~kg} \mathrm{~m}^{-3}$ and $\psi=33^{\circ} . \Delta y$ is $\sim 1 \mathrm{~mm}$ when the fracture begins to propagate within the weak-layer surface hoar. The four particle traces coincide in the figure since accuracy is not good enough to distinguish between them.

Since the experimental data contain elastic and viscous effects, $E^{\prime}$ is defined as the storage modulus in a viscoelastic sense. Calculations of slab deformation using $E^{\prime}$ should yield approximate estimates of the elastic (or recoverable) deformation which is the component responsible for fracture propagation.

In snow fracture applications, an important aspect resides in the experimental information that alpine snow is a quasibrittle material (Bažant and others, 2003). Experimental data (Sigrist, 2006; Borstad and McClung, 2009) from tensile laboratory tests show that alpine snow has a finite-sized fracture process zone (FPZ) which is typically $\sim 5 \mathrm{~cm}$. This implies that the appropriate choice of rate for the material response is governed by the ratio of the rate of fracture advance to the size of the FPZ (Palmer and Rice, 1973; Rice, 1973; Bažant, 2005). For the field tests under snow slabs (propagation saw tests) (Gauthier, 2007; Gauthier and Jamieson, 2008; McClung, 2009a, 2011), the appropriate rate corresponds to a frequency of interest of $\sim 1 \mathrm{~Hz}$ since the saw-blade width $(5 \mathrm{~cm})$ is comparable to the $\mathrm{FPZ}$ width $(5-10 \mathrm{~cm})$ and the saw-cut speed is about $10-20 \mathrm{~cm} \mathrm{~s}^{-1}$.

For a frequency of $1 \mathrm{~Hz}$ (Camponovo and Schweizer, 2001), a value of $E^{\prime}$ from the storage shear modulus in viscoelastic tests may be approximated as

$$
E^{\prime}=2.22 \times 10^{-6}\left[10^{5.58+0.00857(\rho-215)}\right]
$$

In general, the saw tests are too slow to consider the slab deformation as completely elastic (McClung, 2009a, 2011). Thus, in order to estimate the elastic component necessary to drive fractures, we take the storage modulus (the elastic component of the viscoelastic modulus) appropriate for the test rate and the slab density as in Eqn (1). Since the test data contain both elastic and viscous (non-recoverable) deformation, the deformation measurements as in Figure 2 will be overestimates in regard to our calculations below 
from the framework of elasticity since Eqn (1) represents only the elastic part. For the rate of deformation in the experiments, it is not possible that all of the $1 \mathrm{~mm}$ slopenormal displacement prior to dynamic motion is recoverable (elastic) deformation.

\section{PREVIOUS AVALANCHE RELEASE MODELS BASED ON SLAB BENDING}

In this section, we consider the two previous quantitative models based on slab bending. The reader is referred to Perla and LaChapelle (1970), Perla (1971) and Heierli and others (2008) for more details of these previous models.

\subsection{The Perla and LaChapelle (1970) model}

The first quantitative analysis of slab bending prior to avalanche initiation was made by Perla and LaChapelle (1970) and Perla (1971) for application to thick weak layers. In the only example they gave, severe slab bending is implied by strong compressive stresses and bending, which conflicts with our field measurements (Fig. 2) and analysis. The basal boundary conditions are given based on compressive stress perturbations. Their model implies stress singularities within the slab (finite stress change over zero distance). At the time of construction of the model, the failure and fracture properties of alpine snow were unknown, so, of necessity, the model was kept fairly basic. There is no estimate of energy due to bending and there is no fracture mechanics implied by the model. Since the Perla and LaChapelle (1970) model does not contain quantitative estimates of energy or deformation, it is not considered further here since the focus of the present paper is on deformation and energy.

\subsection{The Heierli and others (2008) model}

The only other quantitative model with slab bending and energy specified is given by Heierli and others (2008). In this model, most of the energy input comes from slab bending and slab shear deformation prior to fracture. In Appendix B, the energy formulation for the model of Heierli and others (2008) is given and compared with results from field measurements of $L, D, \rho$ and $\psi$ presented by McClung (2009a, 2011) and as described in Section 3.

The results in Appendix B show that if the slab modulus is chosen as in Eqn (1), appropriate for the rate at which the experiments are conducted, with $\rho=135-262 \mathrm{~kg} \mathrm{~m}^{-3}$, the total energy implied varies between 0.13 and $7.7 \mathrm{~J} \mathrm{~m}^{-2}$ (column 6 in Table 5), i.e. about 4-110 times the fracture energy $\left(0.03-0.07 \mathrm{~J} \mathrm{~m}^{-2}\right)$ used by Heierli and others (2008). Later, Heierli (2008) proposed a mixed-mode fracture energy for use with the model between 0.01 and $0.1 \mathrm{~J} \mathrm{~m}^{-2}$, but this range of values does not change the conclusions in regard to column 6 of Table 5 .

Heierli (2008) gave a modulus as in Eqn (B3), from the mean of data presented by Scapozza (2004) from static triaxial creep tests for use with the model. For the strain rates at which the creep tests were performed $\left(10^{-4}-10^{-6} \mathrm{~s}^{-1}\right.$; Scapozza, 2004), the moduli estimated contain a large component of viscous deformation. Application of this modulus to the measurements in Table 5 gave fracture energies of $0.02-1.2 \mathrm{~J} \mathrm{~m}^{-2}$ (column 7 in Table 5). These values still suggest slab deformation energy input (balanced by fracture energy) which is too high in most cases. The model performance is highly dependent on input of slab modulus, which is a factor of four to eight higher than suggested as appropriate for the rate at which the experiments were conducted (Eqn (1)) for the density range compared. In order to achieve a balance between fracture energy and slab energy for the Heierli and others (2008) model, one is forced to use a very high modulus to reduce the magnitude of the slab-bending and shear-energy terms.

From the perspective of the propagation saw tests, the model physics for both the Perla and LaChapelle (1970) and Heierli and others (2008) models conflicts with our field measurements, observations and analysis which show strong bending and collapse is a dynamic effect occurring after propagation, not before. Our observations from the film results show that collapse and strong bending is a dynamic effect that follows either slope-parallel propagation or slab tensile fracture. Thus, these models seem more appropriate for the dynamic case than the quasi-static case which is the focus of the present paper. The film results and associated field observations of the sequence of events we report are subject to much less uncertainty than any discussion of the appropriate modulus for the tests.

\section{SLAB BENDING ON A DEFORMABLE WEAK LAYER PRIOR TO FRACTURE}

Here we consider the slab-bending effects due to slopenormal deformation from three perspectives considering the slab as a cantilever beam:

1. The maximum implied slope-normal displacement at the downslope, free-surface end of the slab is calculated. We compare the estimated values for the field tests with our measured values (e.g. Fig. 2).

2. The minimum radius of curvature for the beam is calculated for the field shear test results. We then compare the minimum bending radii with minimum radii for three-point beam-bending tensile fracture test data from laboratory results.

3. The implied maximum and mean slab-bending energy per unit area $\left(\mathrm{J} \mathrm{m}^{-2}\right)$ due to slope-normal deformation is calculated. We compare these values with those for mode I fracture energy from previous studies (Sigrist, 2006; McClung, 2007).

For each of the three cases, we consider (a) the case of maximum bending for a free cantilever beam subject to a uniform load of slope-normal stress due to slab weight over the entire beam and unsupported at the bottom; and (b) a uniformly loaded beam supported on an elastic foundation equivalent to a Winkler foundation. Case (a) is important since it represents the situation for maximum bending which is similar to the assumptions in the Perla (1971) and Heierli and others (2008) models. Case (b) is important since some support is suggested by field observations during the propagation saw tests, and it is also important for avalanche release which is the primary application of the work in this paper.

\subsection{Snow slab on a Winkler foundation}

The field experiments described in Section 2 involve the passage of a snow saw through a thin weak layer (typically $1 \mathrm{~cm}$ to several $\mathrm{cm}$ thick) with a thicker cohesive slab on top (typically $20 \mathrm{~cm}$ to $1 \mathrm{~m}$ thick or more). This layering is exactly the same as for the stratigraphy prior to slab 


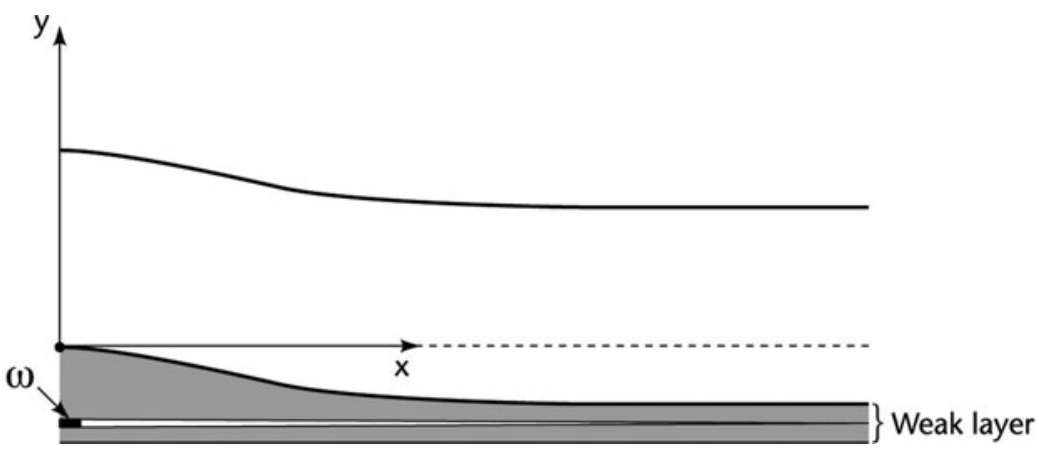

Fig. 3. Schematic of the fracture saw-cut experiments. The saw-cut is made starting from the free surface (from right to left). The saw width is comparable to $\omega$ (Fig. 1). The weak layer is shaded.

avalanche release (McClung and Schaerer, 2006). Field measurements using the saw tests show that if the slab is not cohesive enough, no weak-layer fractures will result. More accurately, there must be a significant increase in hardness of the slab compared to the thin weak layer to obtain a result. As the saw passes, the bottom of the slab remains in contact with the top of the weak layer. With the slab and weak layer in contact, the situation may be thought of as a thicker, cohesive slab on top of a weaker layer which is deformed by saw passage. We approximate this situation considering the slab deforming on a foundation which deforms linearly. The formalism is most well developed by Hetényi (1946), and his approach is followed here.

Hetényi (1946) and Timoshenko (1956) presented applications for the theory of beam bending on an elastic foundation using the assumptions of Winkler (1867). Based on the speed of the cut $\left(20 \mathrm{~cm} \mathrm{~s}^{-1}\right)$ from McClung (2009a, 2011), the saw experiments are too slow to be modelled as purely elastic. However, it is still possible to model the results exploiting the rate dependence exhibited by alpine snow using an effective linear viscoelastic modulus (Eqn (1)) appropriate for the speed of the saw-cut as defined in Section 3.2.

Winkler foundations are routinely applied for soil foundations where the assumptions hold approximately, and for floating ice where the assumptions hold exactly (Bažant and Cedolin, 2003). For alpine snow, the assumptions will be an approximation as with soil applications. Hetényi (1946) stated that a Winkler foundation is mathematically by far the simplest formalism one can make regarding the nature of a linear supporting medium. Any foundation support will imply less bending than without support. Our use of the Winkler foundation is only to show the trend (less bending) when support is in place using the simplest formalism. The Winkler foundation is not proposed as the most realistic type of foundation for the application here.

We list three assumptions to apply Winkler foundation to our test results: (1) the reaction forces in the weak layer at every point are proportional to the deflection of the slab at that point; (2) the weak layer and slab deform only along the portion directly under the loading (where the saw-cut is made); and (3) the lower boundary of the slab is in contact with the upper boundary of the weak layer, but the crack faces within the weak layer may have very weak contact at most locations (Fig. 2). Assumptions 1 and 2 are appropriate for a Winkler foundation. Assumption 3 comes from our field observations made with high-speed films. Figure 3 shows the suggested geometry for assumption 3.
There is some controversy about the amount of contact between the crack faces in the saw test experiments. Our observations and film results indicated weak contact in all cases, whereas Sigrist (2006) reported cases with crack faces out of contact. For this reason, our Winkler analysis includes the unsupported cantilever (faces out of contact) as a limit and the supported cantilever (weak contact).

For modelling the slow-slab bending prior to any fracture, the situation is assumed as in Figure 4. The total slab depth in the slope-normal direction is $D(\mathrm{~m})$. It is assumed that the slope-normal stress on the weak layer due to slab weight is

$$
q=(\rho g \cos \psi) D\left(\mathrm{~N} \mathrm{~m}^{-2}\right)
$$

where $g$ is the magnitude of gravity acceleration $\left(\mathrm{m} \mathrm{s}^{-2}\right)$.

The modulus of the foundation, $k\left(\mathrm{~N} \mathrm{~m}^{-3}\right)$, is taken to represent the weak-layer stiffness in the slope-normal direction after the saw passes through the weak layer. It is presumed to be smaller than the stiffness of the undeformed weak layer or the slab once weak-layer bonds are cut, which allows the weak layer to deform and the slab to bend due to that deformation.

Following the geometry in Figure 3, slow, downward displacement is presumed to start at $x=0$, with total slopenormal displacement increasing to $\Delta y$ after the saw-cut progresses for some distance but prior to dynamic collapse. The modelling is developed for a beam of finite dimensions, which deforms according to classical beam-bending theory

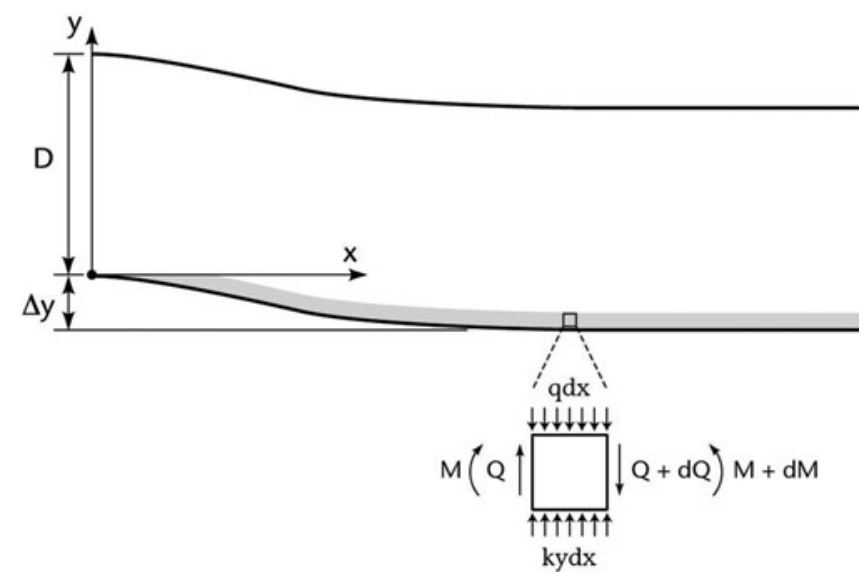

Fig. 4. Schematic of the moment diagram for the bending slab (thickness $D$ ). $q$ is the force per unit area at the slab bottom, $k$ is the foundation modulus and $\Delta y$ is the slope-normal drop. The moment diagram is shown for an infinitesimal element. 
Table 1. Calculations of the total downward slope-normal displacement at the downslope (free surface) end of the slab. The calculations are for 42 slab-weak-layer combinations (559 tests). The results are for the supported slab $(\lambda L=\pi / 2 ; \lambda L=\pi)$ and the unsupported slab $(\lambda \rightarrow 0)$. From Appendix $A$, the median values are the most meaningful

\begin{tabular}{lcc} 
Parameter & $\begin{array}{c}\text { Median } \\
\mathrm{mm}\end{array}$ & $\begin{array}{c}\text { Mean } \\
\mathrm{mm}\end{array}$ \\
\hline$\Delta y_{\lambda L=\pi}$ & 0.03 & 0.1 \\
$\Delta y_{\lambda L=\pi / 2}$ & 0.1 & 0.3 \\
$\Delta y_{\lim \lambda \rightarrow 0}$ & 0.3 & 1.0 \\
\hline
\end{tabular}

developed for the beam supported on a Winkler foundation. The finite beam with support $(k>0)$ represents much less bending than the unsupported beam $(k=0)$ for the same total slope-normal drop, $\Delta y$, at the right end of the beam. From the finite beam solution, in the limit of no support without the crack faces touching $(k \rightarrow 0)$, maximum bending is implied. The solutions with support $(k>0)$ are expected to be more appropriate for the case of avalanche release for which no disturbance, as created by the saw-cut, is expected.

\subsection{Force balance for a beam on a Winkler foundation}

The force balance for a differential beam element on a Winkler foundation generates the slab moments (see Fig. 4). An upward shear force, $Q$ (per unit width), is envisioned at the left end of an infinitesimal element, and downward shear force, $-(Q+d Q)$, is placed at the right end.

The force balance then gives the well-known result (Hetényi, 1946)

$$
Q-(Q+\mathrm{d} Q)+k y \mathrm{~d} x-q \mathrm{~d} x=0\left(\mathrm{~N} \mathrm{~m}^{-1}\right)
$$

By definition, from classical beam-bending theory, with $M$ as the moment and $D_{0}$ as the flexural rigidity, the following expression arises (Hetényi, 1946):

$$
\frac{\mathrm{d}^{2} M}{\mathrm{~d} x^{2}}=\frac{\mathrm{d} Q}{\mathrm{~d} x}=-D_{0} \frac{\mathrm{d}^{4} y}{\mathrm{~d} x^{4}}\left(\mathrm{~N} \mathrm{~m}^{-2}\right)
$$

The flexural rigidity is represented as $D_{0}=E^{\prime} D^{3} / 12$ assuming the neutral axis of the slab (beam) bending is at halfheight, $D / 2$.

Combination of Eqns (2-4) gives the well-known differential equation for the slope-normal deformation for a uniformly loaded ( $q$ : constant) slab sitting on a deformable weak layer (foundation) (Hetényi, 1946):

$$
D_{0} \frac{\mathrm{d}^{4} y}{\mathrm{~d} x^{4}}+k y=q\left(\mathrm{~N} \mathrm{~m}^{-2}\right)
$$

\subsection{Solution for a supported finite beam}

First we consider the slab a cantilever beam of finite length uniformly loaded by the slab weight per unit area, $q$, all along its length. For the calculations below, we begin with the beam supported by a Winkler foundation, and then derive the extreme case of an unsupported beam from the formulation to illustrate the case of maximum bending. In the experiments, the saw-cut made in the weak layer causes the slab (beam) to bend as the cut is made, with maximum slope-normal deformation at the free end where the cut is started. The boundary conditions are taken as fixed (no slope-normal displacement or rotation) at the left end of the beam $(x=0)$ and free at the right end of the beam $(x=L)$. Hetényi (1946) gives the solution to Eqn (5) as

$$
y=-\frac{q}{k}\left[1-\frac{1}{\cosh ^{2}(\lambda L)+\cos ^{2}(\lambda L)}\{A-B\}\right]
$$

where $k=4 D_{0} \lambda^{4}$ and

$$
A=\cosh (\lambda L)[\sin (\lambda x) \sinh \lambda(L-x)+\cos (\lambda x) \cosh \lambda(L-x)]
$$$$
B=\cos (\lambda L)[\sinh (\lambda x) \sin \lambda(L-x)-\cosh (\lambda x) \cos \lambda(L-x)] \text {. }
$$

From Eqn (6), the total downward displacement at $x=L$ is (Hetényi, 1946)

$$
\Delta y=-\frac{q}{k}\left(1-\frac{2 \cosh (\lambda L) \cos (\lambda L)}{\cosh ^{2}(\lambda L)+\cos ^{2}(\lambda L)}\right)
$$

Equations (6) and (7) give the displacements as negative to conform to the coordinate system in Figures 2-4. In the equations below, we have written the solutions in terms of the absolute values of the displacements so that all quantities are expressed as positive. Thus, to conform to the notation and coordinate system used, the replacements $y \rightarrow-y ; \Delta y \rightarrow-\Delta y$ should be made for the equations below.

Further below, we consider the special case of foundation (weak-layer) support for which $\lambda L=\pi / 2$, which from Eqn (7) implies $\Delta y=q / k$ or an effective value

$$
\Delta y_{\lambda L=\pi / 2}=0.49 \frac{q}{E^{\prime}}\left(\frac{L}{D}\right)^{3} L=0.49 \Delta y_{0}
$$

All of our equations below scale with the length parameter, $\Delta y_{0} \equiv\left(q / E^{\prime}\right)(L / D)^{3} L$, from Eqn (8), so this notation is used below. When calculated using the test data, the parameter $\Delta y_{0}$ (42 slab-weak-layer combinations; 559 tests) has a median value of $0.2 \mathrm{~mm}$ and a mean of $0.7 \mathrm{~mm}$, with $75 \%$ of the values less than $1 \mathrm{~mm}$. The parameter $\Delta y_{0}$ is approximately log-normally distributed and the distribution is highly skewed. The probability density function (pdf) of $\Delta y_{0}$ is considered in Appendix A. The analysis in Appendix A implies that the most meaningful estimate of $\Delta y_{0}$ is the median. This is important in the comparison with the experiments below.

\subsection{Limit case for the unsupported cantilever}

For the case of the unsupported beam, in the limit as $\lambda \rightarrow 0$, by multiple application of L'Hospital's rule, Eqns (6) and (7) are respectively (Timoshenko, 1940)

$$
y_{\lim \lambda \rightarrow 0}=\Delta y_{\lim \lambda \rightarrow 0}\left(\frac{2 x^{2}}{L^{2}}-\frac{4 x^{3}}{3 L^{3}}+\frac{x^{4}}{3 L^{4}}\right)
$$

and

$$
\Delta y_{\lim \lambda \rightarrow 0}=\frac{3}{2} \frac{q}{E^{\prime}}\left(\frac{L}{D}\right)^{3} L=\frac{3}{2} \Delta y_{0}
$$

Calculations with Eqns (8) $(\lambda L=\pi / 2)$ and (10) $(\lambda=0)$ for 42 slab-weak-layer combinations (559 tests) are given in Table 1 . The calculated median values $(1 \mathrm{~mm}$ or less) are consistent with our measured values from the field tests. For the calculations, $74 \%$ of the slab-weak-layer combinations gave $\Delta y<1 \mathrm{~mm}$ for the maximum bending case of Eqn (10). Since the calculations are meant to represent the elastic portion only (the elastic storage modulus is used) we suggest the agreement is good. The median values $(0.3 \mathrm{~mm}(\lambda \rightarrow 0)$ 
Table 2. Median, mean and range of $R_{\min }(\mathrm{m})$ calculated from field experiments using Eqns (13) and (14) for 42 slab-weak-layer combinations representing 559 field tests with snow density range $85-266 \mathrm{~kg} \mathrm{~m}^{-3}$

\begin{tabular}{lccc}
\hline Median & Mean & Range & Description \\
\hline 111 & 320 & $16-2748$ & $\lambda L=\pi / 2$ \\
45 & 130 & $6-1117$ & $\lambda \rightarrow 0$ \\
\hline
\end{tabular}

and $0.1 \mathrm{~mm}(\lambda L=\pi / 2))$ are less than our measured values of $\sim 1 \mathrm{~mm}$, which is expected since the tests contain some viscous deformation not included in the elastic calculations (Eqns (8) and (10)). With foundation support $(\lambda>0)$, as would be expected for avalanche initiation, the value of $\Delta y_{\lambda L=\pi / 2}$ over the distance $L$ is very small, implying very little bending. The value $\lambda L=\pi / 2$ can be regarded as an intermediate value for support. For the case of $\lambda L=\pi$, $\Delta y_{\lambda L=\pi}=0.14 \Delta y_{0}$, and the median and mean values (Table 1) yield 0.03 and $0.1 \mathrm{~mm}$ respectively. Thus, from the perspective of the saw-test data and the model here, values for support range from $0 \leq \lambda L \leq \pi$, i.e. from extreme bending $(\lambda=0)$ to negligible bending $(\lambda L=\pi)$. We have no information about what an accurate value of $\lambda$ might be for slab contact and foundation support. In our calculations below, we consider only the cases of extreme bending $(\lambda=0)$ and intermediate support $(\lambda L=\pi / 2)$.

\subsection{Bending estimated for field experiments: minimum radius of curvature}

The minimum radius of curvature, $R_{\min }$, due to slopenormal deformation along the beam is a measure of the maximum degree of bending in the slab prior to fracture.

The curvature of the slab (beam) may be expressed (Bažant and Cedolin, 2003) as

$$
\frac{1}{R}=\frac{y^{\prime \prime}}{\left(1+y^{\prime 2}\right)^{3 / 2}}=y^{\prime \prime}\left(1-\frac{3}{2} y^{\prime 2}+\frac{15}{8} y^{\prime 4}-\ldots\right)
$$

where $y^{\prime \prime}=d^{2} y / d x^{2}$. For the present case, the overall slope of the deflection curve, $y^{\prime}=\mathrm{d} y / \mathrm{d} x$, is small, so the approximation

$$
\frac{1}{R} \approx y^{\prime \prime}
$$

is used. Typically expected values for the scale of slopenormal displacement are $1 \mathrm{~mm}$, and the horizontal scale over which this takes place is several tens of $\mathrm{cm}$, so the mean deflection slope is of order 0.01, which justifies the approximation in Eqn (12).

For the case of the supported beam, Eqns (5) and (12) yield the minimum radius at $x=0$ :

$$
R_{\min (\lambda L=\pi / 2)}=\frac{\pi^{2}}{24} \frac{L^{2}}{\Delta y_{0}}
$$

For the unsupported cantilever beam, evaluation gives

$$
R_{\min (\lim \lambda \rightarrow 0)}=\frac{1}{6} \frac{L^{2}}{\Delta y_{0}}
$$

Table 2 contains calculated values from Eqns (13) and (14) from the field experiments (559 tests; 42 slab-weak-layer combinations). In Table 2, the most meaningful values are the median due to the highly skewed pdf for $\Delta y_{0}$. The
Table 3. Median, mean and range of $R_{\min }(\mathrm{m})$ for laboratory beambending tensile fracture tests for two different load spans with snow density range $149-364 \mathrm{~kg} \mathrm{~m}^{-3}$

\begin{tabular}{lcccc}
\hline Median & Mean & Range & Description & $\begin{array}{c}\text { Number of } \\
\text { tests }\end{array}$ \\
\hline 3 & 3 & $1-8$ & $\begin{array}{c}\text { Span } 0.20 \mathrm{~m} \\
\text { Depth } 0.10 \mathrm{~m} \\
\text { Span } 0.25 \mathrm{~m} \\
\text { Depth } 0.10 \mathrm{~m}\end{array}$ & 65 \\
\hline
\end{tabular}

difference between the median and mean values shows the effect of the highly skewed pdf for $\Delta y_{0}$ (Appendix A).

During winter 2009, we performed three-point beam laboratory bending fracture experiments on small beams of alpine snow in a cold laboratory. The minimum radius of curvature at the instant of tensile fracture (under peak load) was estimated by assuming the snow beams were simply supported (Timoshenko, 1940). Table 3 contains estimates from laboratory tensile three-point bending tests representing the condition at the point of tensile fracture for snow beams with two different load spans: $0.20 \mathrm{~m}$ and $0.25 \mathrm{~m}$, implying span-to-depth ratios $2: 1$ and $2.5: 1$.

Comparing the results (Tables 2 and 3), the median of $R_{\min }$ for either the median of supported $(\lambda L=\pi / 2)$ or unsupported $(\lambda=0)$ beam exceeds the median values for the laboratory tensile fracture tests by approximately a factor of ten and five respectively. Tables 2 and 3 lead to the conclusion that the laboratory tensile tests (representing the tensile fracture condition) show more bending than the field tests. These results agree with our observations of the field tests since tensile fracture in the field tests is observed only after the slab is in a dynamic condition following weak-layer collapse (large slope-normal displacement) due to slopeparallel propagation or after a cut is made with a thick saw. There is no denying that slab bending takes place in the experiments given that slab tensile fractures sometimes occur prior to slope-parallel propagation in the weak layer when a thick saw is used. However, extrapolation to the case of avalanche release cannot be directly made due to the artificial slope-normal displacement introduced by making the saw-cut in the tests.

\subsection{Mean slab and maximum slab strain bending work per unit area}

Another method to illustrate bending is to calculate the slab strain energy of bending per unit area. The differential strain work to bend a segment, $\mathrm{d} s$, of arc length measured along the neutral axis of a cylindrical beam is (Fung, 1965)

$$
\mathrm{d} U=\frac{1}{2} D_{0} \frac{\mathrm{d} s}{R^{2}}
$$

Assuming that deflection of the beam is infinitesimal, with $\mathrm{d} s$ approximated by $\mathrm{d} x$, the total strain energy over a length, $L$, per unit width is given by (e.g. Fung, 1965)

$$
U=\frac{1}{2} \int_{0}^{L} D_{0}\left(\frac{\mathrm{d}^{2} y}{\mathrm{~d} x^{2}}\right)^{2} \mathrm{~d} x(\mathrm{~N})
$$

From Eqn (16), the mean work of bending over the distance $L$ 
Table 4. Median values for expected limits: upper (maximum) and lower (mean) bending energy $\left(\mathrm{J} \mathrm{m}^{-2}\right)$ for 42 slab-weak-layer combinations representing 559 tests. The values may be compared with the mode I slab fracture energy of $0.1-1 \mathrm{~J} \mathrm{~m}^{-2}$ estimated by Sigrist (2006) and McClung (2007)

Strain energy parameter

Median value

$\begin{array}{ll}\bar{W}_{\lambda L=\pi / 2} & 0.003 \\ W_{\max (\lambda L=\pi / 2)} & 0.025 \\ \bar{W}_{\lambda \rightarrow 0} & 0.03 \\ W_{\max (\lambda \rightarrow 0)} & 0.15\end{array}$

per unit area is given by

$$
\bar{W}=\frac{1}{2} \frac{1}{L} \int_{0}^{L} D_{0}\left(y^{\prime \prime}\right)^{2} \mathrm{~d} x\left(\mathrm{~J} \mathrm{~m}^{-2}\right)
$$

Application of Eqn (17) for the displacement profile of Eqn (5) gives the following result for the supported beam:

$$
\bar{W}_{\lambda L=\pi / 2}=0.034\left(q \Delta y_{0}\right)\left(\mathrm{J} \mathrm{m}^{-2}\right)
$$

A similar calculation for the unsupported beam gives

$$
\bar{W}_{\lim \lambda \rightarrow 0}=0.3\left(q \Delta y_{0}\right) \quad\left(\mathrm{J} \mathrm{m}^{-2}\right)
$$

From Eqn (16), with use of Eqns (13) and (14), a maximum estimate of bending strain energy at the left end (tip) of the supported beam is given by

$$
W_{\max (\lambda L=\pi / 2)}=0.25 q \Delta y_{0}\left(\mathrm{~J} \mathrm{~m}^{-2}\right)
$$

and for the unsupported beam the result is:

$$
W_{\max (\lambda \rightarrow 0)}=\frac{3}{2} q \Delta y_{0}\left(\mathrm{~J} \mathrm{~m}^{-2}\right)
$$

Table 4 shows median values calculated from Eqns (18-21). We expect that the average value $(\bar{W})$ applies to a lower bound and the maximum value $\left(W_{\max }\right)$ approximates an upper bound. For a quasi-brittle material like snow, the average values over some suitable length scale (e.g. the fracture process zone) would seem most appropriate, rather than the point values at the tip (left end of the beam). Thus, we would expect the maximum values to be too high. The maximum values are generally in the lower range or below the mode I fracture energy estimated by Sigrist (2006) and McClung (2007) which is in the range $0.1-1 \mathrm{~J} \mathrm{~m}^{-2}$. Based on the results (Table 4) it seems possible that bending energy can contribute to tensile fracture generation at or near the saw-cut tip. It is beyond the scope of the present paper to discuss such tensile fracture generation.

The introduction to the calculations of length $L$ has two important implications. One, as considered here, is to enable estimates of bending of the slab modelled simply as a beam given the maximum value of $L$ at the condition of propagation. The other is to consider the critical length when the propagation condition within the weak layer is met (McClung, 2009a, 2011), which is beyond the scope of the present paper.

Lack of contact over some portion of the crack-tip faces is crucial for the anti-crack model of Heierli and others (2008). If a fracture-mechanical-type approach is adopted, it implies a negative mode I stress intensity factor. In the original paper on anti-crack formation (Fletcher and Pollard, 1981), the lack of contact or inner penetration of the faces is by pressure solution and diffusion processes. There is no such mechanism for dry snow. Precision experimental data from laboratory shear tests (McClung, 2009a) for faceted snow show that, in displacement-controlled shear experiments, faceted snow is in a dilatant state, which implies fracture occurs due to slip surface formation by grains riding up over each other.

Reiweger and Schweizer (2010) performed both loadand displacement-controlled precision shear fracture tests on a surface hoar monocrystal layer between two layers of rounded grains. Their results showed that, at the time of fracture, $\sim 90 \%$ of the deformation was in shear within the surface hoar layer, with small or negligible compressive (or compactant) slope-normal deformation.

The laboratory shear test results (from both faceted and surface hoar) are extremely important since they suggest that the slope-normal (bending) deformation seen in the saw tests prior to fracture is due to the disturbance created by the saw, as stated above. Any lack of contact during the saw test experiments is most likely due to the artificial gap or disturbance created by the saw-cut, with an unlikely relevance to natural avalanche release.

More than $80 \%$ of the slab-weak-layer combinations used in this paper are from faceted and surface-hoar weak layers which are the primary weak layers (called persistent layers) observed to collapse dynamically in field observations.

\section{LIMITATIONS AND DISCUSSION}

Our results are developed from a combination of input estimates from a variety of sources including laboratory measurements, field measurements, estimates from avalanche fractures and films of field tests. Although desirable, it would be nearly impossible to measure everything necessary for input to the bending calculations. It is extremely important to use a modulus which is appropriate to the rate and density dependence for the test results and consistent with the quasi-brittle character of alpine snow. The modulus was not measured in the experiments, so the analytical results derived are dependent on Eqn (1) which represents the elastic (storage) modulus appropriate for the test rate and density derived from Camponovo and Schweizer (2001). The modulus (Eqn (1)) takes into account rate and density, but also varies with snow structure, so this formulation is subject to some uncertainty.

It is not necessary to do any elastic modelling calculations to show that bending prior to slope-parallel weak-layer propagation is small. If one takes the total measured slopenormal displacement, $\Delta y=0.001 \mathrm{~mm}$, assuming the radius of curvature is constant (on a circle), the radius may be approximated as $R \approx L^{2} / \Delta y$. Calculations (68 slab-weaklayer combinations; 750 tests) gave median $96 \mathrm{~m}$, mean $126 \mathrm{~m}$ and range $5-423 \mathrm{~m}$. These should be regarded as minimum values since the deformation measured, $\Delta y$, will certainly not be entirely elastic. The values are consistent with those in Table 1 calculated from the estimated elastic portion of the deformation.

Some of the results depend on the assumptions of a Winkler foundation. It is known that for soils the Winkler foundation is only approximate, and we expect the same for alpine snow. For the application here, the snow is assumed dry snow and the test results are all for dry snow. This may be an advantage over soil applications, which typically include the effects of water.

We have not dealt with slope-parallel weak-layer deformation in this paper. The fact that the first fracture to propagate 
in most cases in the saw tests is in the slope-parallel direction suggests the weak-layer deformation component in that direction is important. In the saw test experiments, the bonds in the slope-parallel direction are cut by the saw. Since there is measurable slope-normal deformation along with the tests, it is likely the weak-layer deformation pattern is two-dimensional (2-D). It is beyond the scope of the present paper to model the 2-D deformation pattern. The work of Reiweger and Schweizer (2010) confirms that, for surface hoar, the slope-parallel component of deformation is responsible for the weak-layer fracture without the disturbance and slope-normal component of deformation created by the saw-cuts in the field tests. Even if the deformation pattern is regarded as 2-D, this would not change the sequence of events revealed and slope-normal deformation in the films which is the primary source of evidence here. Similar comments apply to any slab rotation near the tip of the saw-cut. We have no measurements to estimate the effects of slab rotation, but this would not affect the film results including the sequence of events.

\section{SUMMARY}

The problem of slope-normal deformation within weak layers of finite thickness for dry-snow slab avalanche failures has been modelled twice before, first by Perla and LaChapelle (1970) and Perla (1971) and recently by Heierli and others (2008). Perla's model contains no fracture mechanics. It is based on field observations of avalanches releasing, which reveal dynamic collapse taking place during rapid fracture propagation, but the assumption is made that this also occurs prior to propagation.

The latest model (Heierli and others, 2008) rests on assumptions similar to those of Perla and LaChapelle (1970), with implicit assumptions of strong slab bending and slab shear deformation prior to fracture propagation. At present, there are no reported measurements of weak-layer deformation along with the propagation saw tests prior to propagation. However, Reiweger and Schweizer (2010) reported data for shear failure of a surface hoar layer between two layers of similar hardness without a notch or disturbance as caused by the saw. Their results showed that $\sim 90 \%$ of the deformation was shear deformation in the surface hoar layer and that shear deformation, not slope-normal deformation, was responsible for the fracture. For surface hoar, they showed that the deformation is highly anisotropic, being extremely prone to slope-parallel (shear) deformation and resistant to slope-normal deformation at the instant fracture takes place. Their results are consistent with the observations and analysis in this paper.

The Heierli and others (2008) model is based on concepts of fracture mechanics developed by A.A. Griffith in the 1920s with an infinitesimal fracture process zone (FPZ). An infinitesimal FPZ for alpine snow is refuted by the tests of Sigrist (2006) and Borstad and McClung (2009). Bažant and Planas (1998) showed that a quasi-brittle material such as snow cannot be adequately described with only one fracture parameter such as a fracture energy as modelled by Heierli and others (2008).

Heierli and others (2008) assume that alpine snow is a linear elastic solid, and verification of the model is claimed on the basis of the propagation saw tests modelled assuming the slab is linear elastic. However, the deformation rate in the experiments is much too low for the slab to be considered elastic. It is of interest that if the recommended modulus (Eqn (B3)) is used in the bending calculations in this paper, the median value is $\Delta y_{0}=0.03 \mathrm{~mm}$ (42 slab-weaklayer combinations; 559 tests). Thus, the radii in Table 2 increase by a factor of 6.35 , the energies in Table 4 decrease by a factor of 6.35 and bending effects are largely negligible. In Appendix B, it is shown from field measurements that even with a high-rate $(100 \mathrm{~Hz})$ elastic modulus, the slab energy in the model of Heierli and others (2008) exceeds the recommended values of fracture energy in some cases. In Appendix B it is shown that if account is taken of the rate dependence of slab deformation appropriate for the saw tests (Eqn (1)), the energy for slab bending and shear deformation in Heierli and others (2008) is one to two orders of magnitude higher than required to balance the assumed fracture energy of alpine snow in some cases. The result suggests that the model predicts shorter critical lengths than shown in field measurements in some cases.

The emphasis in this paper is to consider stress and deformation conditions prior to fracture propagation for weak layers of finite thickness. The most compelling evidence comes from the high-speed films combined with particle tracking of the deformation process prior to and after fracture propagation in the saw test experiments and the sequence of events prior to slope-parallel weak-layer fracture propagation. These experiments show that slope deformation prior to fracture propagation is minor $(\mathrm{O}(1 \mathrm{~mm}))$ over long cut distances on the order of $0.5 \mathrm{~m}$ or more. The films also show that severe bending and weak-layer collapse is a dynamic effect that follows fracture propagation. The scale of the vertical deformation over the distances observed ( $>0.5 \mathrm{~m}$ ) provides enough evidence that slab-bending energy is very small or negligible. Thus, the principal argument about the amount of slab bending and deformation does not lie in specification of the proper choice of modulus for use with the propagation saw tests. Instead, it is based on the slope-normal deformation measured prior to slope-parallel propagation and, most importantly, the sequence of events seen in the high-speed films.

The modelling in this paper is based on the assumption of the slab deforming on a weak foundation (the weak layer). With its simple assumptions, the Winkler foundation is popular for the earth materials (soils and floating ice). The Winkler approximation here is the first application for alpine snow. However, the main results in this paper do not depend on the assumptions of the Winkler foundation. The approach here has been to use field data to analyze the problem from the perspective of the limits of maximum bending (no support). The physical principle used is important because it implies that with support $(\lambda>0)$, as expected for avalanche initiation and our experiments, bending effects will be less. The same physical principle will hold for more generalized foundation models such as Wieghardt's foundation as discussed by Bažant and Cedolin (2003). Thus, simple extrapolation to the case of snow slab avalanche initiation from the no-support limit using this principle will hold for more complex foundation assumptions as well. The physical principle implies less bending for the case of avalanche initiation or for the saw test than for the case of the unsupported cantilever, regardless of foundation assumptions.

Our high-speed films also show some important results concerning the dynamic case, after propagation begins in the weak layer. They show dynamic collapse, which closes the crack faces and implies inner penetration of the crack 
faces, with no crack opening, as shown by Van Herwijnen and Heierli (2009). In the saw tests, the saw produces a disturbance behind the crack tip, which sometimes causes bending and tensile fracture of the slab before dynamic propagation. However, observations of avalanche dimensions cast doubt on whether the same concept applies to avalanche release. In the case of avalanches, slope-parallel slab dimensions prior to the appearance of slab tensile fracture upslope are on average about 50D (McClung, 2009b), which is much larger than in the saw test experiments $(\approx 2 D)$. Even though dynamic weak-layer collapse undoubtedly takes place in some cases for avalanche release, the physical processes prior to propagation may be considerably different from those in the saw tests. Namely, there may be no disturbance similar to that created artificially in the saw tests. Bažant and others (2003) proposed that strain and stress concentrations within the weak zone are related to mode II fracture initiation. However, no disturbance (with crack faces out of contact) is needed for that mechanism.

\section{ACKNOWLEDGEMENTS}

This work was supported by the Natural Sciences and Engineering Research Council of Canada, Canadian Mountain Holidays and the University of British Columbia. We are very grateful for their support.

\section{REFERENCES}

Bažant ZP (2005) Scaling of structural strength, 2nd edn. Elsevier Butterworth Heinemann, Oxford

Bažant ZP and Cedolin L (2003) Stability of structures: elastic, inelastic, fracture and damage theories. Dover Publications, Mineola, NY

Bažant ZP and Planas J (1998) Fracture and size effect in concrete and other quasibrittle materials. CRC Press, Boca Raton, FL

Bažant ZP, Zi G and McClung D (2003) Size effect law and fracture mechanics of the triggering of dry snow slab avalanches. J. Geophys. Res., 108(B2), 2119 (doi: 10.1029/2002JB001884)

Borstad CP and McClung DM (2009) Size effect in dry snow slab tensile fracture. In Proceedings of the 12th International Conference on Fracture, 12-17 July 2009, Ottawa, Ontario, Canada. National Research Council, Canada, Ottawa

Camponovo C and Schweizer J (2001) Rheological measurements of the viscoelastic properties of snow. Ann. Glaciol., 32, 44-50 (doi: 10.3189/172756401781819148)

Fletcher RC and Pollard DD (1981) Anticrack model for pressure solution surfaces. Geology, 9(9), 419-424 (doi: 10.1130/00917613(1981)9<419:AMFPSS>2.0.CO;2)

Fung YC (1965) Foundations of solid mechanics. Prentice Hall, Engelwood Cliffs, NJ

Gauthier D (2007) A practical field test for propagation and arrest in weak snowpack layers in relation to slab avalanche release. (PhD thesis, University of Calgary)

Gauthier D and Jamieson JB (2008) Evaluation of a prototype field test for fracture and failure propagation in weak snow pack layers. Cold Reg. Sci. Technol., 51(2-3), 87-97 (doi: 10.1016/ j.coldregions.2007.04.005)

Heierli J (2008) Anticrack model for slab avalanche release. (PhD thesis, University of Karlsruhe)

Heierli J, Gumbsch P and Zaiser M (2008) Anticrack nucleation as triggering mechanism for snow slab avalanches. Science, 321(5886), 240-243 (doi: 10.1126/science.1153948)

Hetényi M (1946) Beams on elastic foundations: theory with applications in the fields of civil and mechanical engineering, University of Michigan Press, Ann Arbor, MI
Johnson BC, Jamieson B and Stewart R (2004) Seismic measurement of fracture speed in a weak snowpack layer. Cold Reg. Sci. Technol., 40(1-2), 41-45 (doi: 10.1016/j.coldregions.2004. 05.003)

McClung DM (1979) Shear fracture precipitated by strain softening as a mechanism of dry slab avalanche release. J. Geophys. Res., 84(B7), 3519-3526

McClung DM (1981) Fracture mechanical model of dry slab avalanche release. J. Geophys. Res., 86(B11), 10 783-10 790

McClung DM (2007) Fracture energy applicable to dry snow slab avalanche release. Geophys. Res. Lett., 34(2), L02503 (doi: 10.1029/2006GL028238)

McClung DM (2009a) Dry snow slab quasi-brittle fracture initiation and verification from field tests. J. Geophys. Res., 114(F1), F01022 (doi: 10.1029/2007JF000913)

McClung DM (2009b) Dimensions of dry snow slab avalanches from field measurements. J. Geophys. Res., 114(F1), F01006 (doi: 10.1029/2007JF000941)

McClung DM (2011) Analysis of critical length measurements for dry snow slab weak-layer shear fracture. J. Glaciol., 57(203), 557-566 (doi: 10.3189/002214311796905541)

McClung D and Schaerer P (2006) The avalanche handbook, 3rd edn. The Mountaineers, Seattle, WA

Mellor M (1975) A review of basic snow mechanics. IAHS Publ. 114 (Symposium at Grindelwald 1974 - Snow Mechanics), 251-291

Palmer AC and Rice JR (1973) The growth of slip surfaces in the progressive failure of over-consolidated clay. Proc. $R$. Soc. London, Ser. A, 332(1591), 527-548

Perla R (1971) The slab avalanche. (PhD thesis, University of Utah) Perla RI and LaChapelle ER (1970) A theory of snow slab failure. J. Geophys. Res., 75(36), 7619-7627

Reiweger I and Schweizer J (2010) Failure of a layer of buried surface hoar. Geophys. Res. Lett., 37(24), L24501 (doi: 10.1029/ 2010GL045433)

Rice JR (1973) The initiation and growth of shear bands. In Palmer AC ed. Proceedings of the Symposium on the Role of Plasticity in Soil Mechanics, 13-15 September 1973, Cambridge, UK. Engineering Department, University of Cambridge, Cambridge, 263-278

Salm B (1977) Snow forces. J. Glaciol., 19(81), 67-100

Scapozza C (2004) Entwicklung eines dichte- und temperaturabhängigen Stoffgesetzes zur Beschreibung des viskoelastischen Verhaltens von Schnee. (PhD thesis, ETH Zürich)

Schweizer J, Jamieson JB and Schneebeli M (2003) Snow avalanche formation. Rev. Geophys., 41(4), 1016 (doi: 10.1029/ 2002RG000123)

Sigrist C (2006) Measurement of fracture mechanical properties of snow and application to dry snow slab avalanche release. (PhD thesis, ETH, Zürich)

Timoshenko S (1940) Strength of materials, Part I. Elementary theory and problems. D. van Nostrand, New York

Timoshenko S (1956) Strength of materials, Part II. Advanced theory and problems, 3rd edn. Krieger Publishing, Malabar, FL

Van Herwijnen A and Heierli J (2009) Measurement of crack-face friction in collapsed weak snow layers. Geophys. Res. Lett., 36(23), L23502 (doi: 10.1029/2009GL040389)

Winkler E (1867) Die Lehre von elastizität und festigkeit mit besonderer Rücksicht auf ihre Anwendung in der Technik für polytechnische Schulen, Bauakademien, Ingenieure, Maschinenbauer, Architecten, etc. H. Dominicus, Prague

\section{APPENDIX A: PROBABILITY DENSITY FUNCTION FOR THE SCALING PARAMETER $\Delta y_{0}$}

All the equations in our bending analysis scale with the parameter $\Delta y_{0} \equiv\left(q / E^{\prime}\right)(L / D)^{3} L$. We fit the 42 calculated values (559 tests) of $\Delta y_{0}$ to numerous pdfs and we found the most suitable pdf is log-normal, with scale parameter 1.61 
and location parameter -1.41 . Three goodness-of-fit parameters were determined: (1) the Kolmogorov-Smirnov statistic is 0.10 , with $p$-value 0.78 (critical value for $\alpha=0.2$ is 0.16 ); (2) the Anderson-Darling statistic is 0.39 (critical value for $\alpha=0.2$ is 1.37); and (3) the chi-squared statistic with 4 degrees of freedom is 2.72 , with $p$-value 0.60 (critical value for $\alpha=0.2$ is 5.99 ).

The sample statistics showed that the pdf for $\Delta y_{0}$ is highly skewed. The ratio of the skewness to standard error of the skewness is 6.80 , and the ratio of the kurtosis to the standard error of the kurtosis is 9.1 . The median of $\Delta y_{0}$ is $0.20 \mathrm{~mm}$ and the mean is $0.69 \mathrm{~mm}$, with a range from 0.001 to $5.07 \mathrm{~mm}$. Due to the highly skewed pdf, the most meaningful estimate of $\Delta y_{0}$ is the median value. Use of the median value for $\Delta y_{0}$ in Eqns (7) and (9) gives $\Delta y_{\lambda L=\pi / 2}=0.1 \mathrm{~mm}$ (supported beam) and $\Delta y_{\lim \lambda \rightarrow 0}=0.3$ $\mathrm{mm}$ (unsupported cantilever). These are very reasonable values since the total measured values of $\Delta y \approx 1 \mathrm{~mm}$ include both elastic and viscous deformation, so they should exceed the values of Eqns (7) and (9) estimated for the elastic component alone.

Similar comments apply to $q \Delta y_{0}$ for the energy calculations. The ratio of skewness to its standard error is 7.7, and the ratio of kurtosis to its standard error is 11.3. Thus, the median is the most meaningful value.

\section{APPENDIX B: ENERGY ANALYSIS OF AN ALTERNATE SLAB DEFORMATION MODEL: HEIERLI AND OTHERS (2008)}

Heierli and others (2008) published a model to explain fracture initiation for snow slabs by proposing that slab bending and slab shearing prior to fracture constitute the major energy inputs when there is slope-normal deformation. In this appendix, comparison of the energy implied by the model is given for nine cases of field-measured median initiation lengths, $L$, derived from 115 separate tests for different slope angles. Measured quantities include $L, D, \rho$ and $\psi$, and estimated quantities include Young's modulus, $E$, for the slab and fracture energy $\left(\mathrm{J} \mathrm{m}^{-2}\right)$, denoted here as $G_{\mathrm{f}}$ and described by Heierli and others (2008) as mixed mode. The model contains two other constants, one of which is a Dundur's elastic mismatch parameter and the other a correction factor assuming a rectangular Timoshenko beam relating $\mu$ and $E$. Both of these are assumed to equal 1 in the comparison below as suggested by Heierli and others (2008) if $v=0.2$.

Let $\sigma=-\rho g D \cos \psi ; \tau=\rho g D \sin \psi$ (Heierli and others, 2008), then the fracture energy is given by

$$
G_{\mathrm{f}}=T_{0}+T_{1}+T_{2}+T_{3}\left(\mathrm{~J} \mathrm{~m}^{-2}\right)
$$

where

$$
\begin{aligned}
& T_{0}=\frac{\left(\sigma^{2}+\tau^{2}\right)}{E}(1.57 L) \\
& T_{1}=\frac{\sigma^{2} L}{E}\left[1.5\left(\frac{L}{D}\right)+1.5\left(\frac{L}{D}\right)^{2}+1.5\left(\frac{L}{D}\right)^{3}\right] \\
& T_{2}=\frac{\tau^{2} L}{E}\left[0.75+0.5\left(\frac{L}{D}\right)+1.5\left(\frac{D}{L}\right)\right] \\
& T_{3}=\frac{ \pm|\sigma \tau| L}{E}\left[3+2.25\left(\frac{L}{D}\right)\right]
\end{aligned}
$$

For $T_{3}$ in Eqn (B2), the plus sign refers to cases where the saw-cut is made upslope and the minus sign to cases for which it is made downslope.

Heierli and others (2008) prescribed $G_{\mathrm{f}}$ as $0.03 \mathrm{~J} \mathrm{~m}^{-2}$ for decomposing and fragmented forms in the weak layer and $0.07 \mathrm{~J} \mathrm{~m}^{-2}$ for weak-layer facets. Inspection of Eqns (B1) and (B2) shows that from the field measurements everything on the right-hand side except $E$ may be determined (measured or calculated). Table 5 gives the field measurements along with $G_{f}$ (column 6) calculated from Eqn (B1) with $E=2 \mu(1+v), v=0.2$ and $\mu$ appropriate for the rate at which the experiments were conducted as from Eqn (1).

Using the maximum value for fracture energy assumed by Heierli and others (2008) $\left(0.07 \mathrm{~J} \mathrm{~m}^{-2}\right)$, the values in Table 5 suggest that the calculated slab energy values exceed their estimates by a factor of 2-160. However, the last three cases in Table 5 were analyzed by Heierli and others (2008) and they assumed a lower value for these $\left(0.03 \mathrm{~J} \mathrm{~m}^{-2}\right)$, so a range of $4-160$ is more appropriate, for which the calculations exceed the fracture energy estimates used.

Heierli (2008) specified an elastic modulus, $E$, appropriate for the model from the work of Scapozza (2004). This work is based on triaxial static creep tests with strain rates in the range $10^{-4}-10^{-6} \mathrm{~s}^{-1}$. It is not possible that such rates

Table 5. Field measurements and calculated fracture energy, $G_{\mathrm{f}}$, from Eqns (B1) and (B2). The values of $G_{\mathrm{f}}$ are those with modulus

\begin{tabular}{|c|c|c|c|c|c|c|c|}
\hline Case & Number of tests & $\begin{array}{c}\rho \\
\mathrm{kg} \mathrm{m}^{-3}\end{array}$ & $\begin{array}{l}L \\
\mathrm{~m}\end{array}$ & $\begin{array}{l}D \\
\mathrm{~m}\end{array}$ & $\psi$ & $\begin{array}{c}G_{\mathrm{f}}(\operatorname{Eqn}(1)) \\
\mathrm{J} \mathrm{m}^{-2}\end{array}$ & $\begin{array}{c}G_{\mathrm{f}}(\operatorname{Eqn}(\mathrm{B} 3)) \\
\mathrm{J} \mathrm{m}^{-2}\end{array}$ \\
\hline 1 & 10 & 177 & 0.43 & 0.37 & 0 & 3.2 & 0.47 \\
\hline 2 & 10 & 185 & 0.61 & 0.30 & 32 & 7.7 & 1.2 \\
\hline 3 & 10 & 146 & 0.19 & 0.34 & 6.5 & 0.70 & 0.09 \\
\hline 4 & 10 & 138 & 0.39 & 0.36 & 38 & 3.9 & 0.50 \\
\hline 5 & 17 & 262 & 0.40 & 0.98 & 0 & 2.8 & 0.64 \\
\hline 6 & 14 & 233 & 0.38 & 1.30 & 23 & 4.3 & 0.87 \\
\hline 7 & 23 & 135 & 0.13 & 0.14 & 0 & 0.13 & 0.02 \\
\hline 8 & 17 & 168 & 0.19 & 0.11 & 30 & 0.28 & 0.04 \\
\hline 9 & 4 & 168 & 0.22 & 0.10 & 38 & 0.38 & 0.06 \\
\hline
\end{tabular}
appropriate for the rate of the experiments, $G_{f}(\operatorname{Eqn}(1))$, and $G_{f}(\operatorname{Eqn}(B 3))$, calculated with the modulus from eqn (B3) of Heierli (2008). Cases 1-4 represent surface-hoar weak-layer forms, cases 5 and 6 are for faceted forms and cases 7-9 represent decomposing and fragmented forms. The cuts on slopes were made in the upslope direction except for case 6 . The values of $L$ are the median for each set of tests 
would produce mostly elastic deformation in alpine snow. Camponovo and Schweizer (2001) showed that at a rate of $1 \mathrm{~s}^{-1}$ there is still viscous deformation. The Young's modulus recommended by Heierli (2008) is

$$
E=0.2 \exp (\rho / 67)(\mathrm{MPa})
$$

Using Eqn (B3), the values of $G_{\mathrm{f}}$ (column 7) in Table 5 range from 0.02 to $1.2 \mathrm{~J} \mathrm{~m}^{-2}$, with a mean of $0.4 \mathrm{~J} \mathrm{~m}^{-2}$, in spite of the fact that Heierli (2008) states that $G_{\mathrm{f}}$ should range from 0.01 to $0.1 \mathrm{~J} \mathrm{~m}^{-2}$, with a mean of $0.04 \mathrm{~J} \mathrm{~m}^{-2}$. Use of this proposed modulus is equivalent to boosting the value of $E$ by a factor of $\sim 7$ over that appropriate for the rate at which the tests were performed. For the last three cases, the results (column 7) show that $G_{f}$ would have to be increased by a factor of 3.5 in order to match the measured values of $L$ using the model, even though the experiments were all done on the same day with the same weak layer.
Gauthier and Jamieson (2008) measured $L=0.40 \mathrm{~m}$ (median of 17 tests; case 5) and a weak layer of faceted forms. Using Eqn (1) for the shear modulus and $G_{\mathrm{f}}=0.07 \mathrm{~J} \mathrm{~m}^{-2}$ for faceted forms implies $L=0.015 \mathrm{~m}$ compared to the measured value of $L=0.40 \mathrm{~m}$. Using a high-rate elastic modulus $(100 \mathrm{~Hz})$ from Sigrist $(2006$, p. 76 , eqn 4.8) implies $L=0.15 \mathrm{~m}$. In order to achieve the measured value $(L=0.40 \mathrm{~m})$ with the assumed fracture energy, model input of $E \approx 100 \mathrm{MPa}$ is necessary, which is not believable for the measured slab density.

For a similar set of measurements, on the same faceted weak layer, with saw-cuts made in the downslope direction, Gauthier and Jamieson (2008) measured $L=0.38 \mathrm{~m}$ (median of 14 tests; case 6$)$. Even with the high-rate elastic modulus $(100 \mathrm{~Hz})$, the model prediction is $L<0.001 \mathrm{~m}$ in this case. The value of $L$ is on the order of one grain in size, which would imply snow could not build up on slopes.

MS received 21 January 2011 and accepted in revised form 12 January 2012 